This item was submitted to Loughborough's Research Repository by the author.

Items in Figshare are protected by copyright, with all rights reserved, unless otherwise indicated.

\title{
Enhanced situational awareness for unmanned aerial vehicle operating in terminal areas with circuit flight rules
}

PLEASE CITE THE PUBLISHED VERSION

https://dx.doi.org/10.1177/0954410016636156

\section{PUBLISHER}

(c) The Author. Published by SAGE

\section{VERSION}

AM (Accepted Manuscript)

\section{PUBLISHER STATEMENT}

This work is made available according to the conditions of the Creative Commons Attribution-NonCommercialNoDerivatives 4.0 International (CC BY-NC-ND 4.0) licence. Full details of this licence are available at: https://creativecommons.org/licenses/by-nc-nd/4.0/

\section{LICENCE}

CC BY-NC-ND 4.0

\section{REPOSITORY RECORD}

Liu, Cunjia, Matthew Coombes, Baibing Li, and Wen-Hua Chen. 2016. "Enhanced Situational Awareness for Unmanned Aerial Vehicle Operating in Terminal Areas with Circuit Flight Rules". figshare.

https://hdl.handle.net/2134/20479. 


\title{
Enhanced situational awareness for UAV operating in terminal areas with circuit flight rules
}

\author{
Cunjia Liu ${ }^{* 1}$, Matthew Coombes ${ }^{1}$, Baibing Li $^{2}$, and Wen-Hua Chen ${ }^{1}$ \\ ${ }^{1}$ Department of Aeronautical and Automotive Engineering, Loughborough University, \\ $\mathrm{UK}$ \\ ${ }^{2}$ School of Business and Economics, Loughborough University, UK
}

December 1, 2015

\begin{abstract}
This paper considers the situational awareness function associated with an Unmanned Aerial Vehicle (UAV) arriving at an uncontrolled airfield. Given no air traffic control service available within such a terminal area, the UAV needs to establish a good level of situation awareness by using its onboard sensors to detect and track other traffic aircraft. Comparing to existing works which mainly use sensor observations in the filtering process, this paper exploits the circuit flight rules to provide extra knowledge about the target behaviour. This is achieved by using the multiple models to describe the target motions in different flight phases and characterising the phase transition in a stochastic manner. Consequently, an interacting multiple model particle filter with state-dependent transition probabilities is developed to provide the required situation awareness function.
\end{abstract}

\section{Introduction}

With the advantages of using Unmanned Aerial Vehicles (UAVs) in areas such as search and rescue, border security, law enforcement, aerial photography and environmental monitoring and the associated commercial implications of those activities, there is a large drive for UAVs to be integrated within the national airspace system [1]. However, there are also a number of requirements and challenges associated with this process to guarantee the safety and coherency of this integration. For example, Civil Aviation Authority U.K. has stated in CAP722 that "For a UAV to operate within busy civil airspace it is required to comply with Air Traffic Control (ATC) instruction in the same way and within the same timeframe that the pilot of a manned aircraft would" [2]. This means that a UAV must be predictable, communicative, perform self-separation and see-and-avoid against other air traffics. These requirements become more critical when a UAV is flying into a terminal area around an airport with high traffic density, which in turn necessitates the development of advanced situation awareness for the UAV.

Situation awareness is the ability of a system to both perceive its environment and make inferences about its future states [3]. In the context of the terminal area operation, this means

\footnotetext{
${ }^{*}$ c.liu5@lboro.ac.uk
} 
that a UAV is able to locate and to predict where other traffic aircraft are at the present and will be in the future, so that the UAV can maintain an acceptable separation from these aircraft, comply with the rules of the air and on an even higher level flies as efficiently as possible [4]. To enable this function, a UAV first needs to locate the traffic aircraft by combining information perceived from different sources such as onboard sensors (optical/radar systems), transponders and even radio telemetry. Then, it needs to comprehend the aircraft's intention indicated by the phase of flights (e.g. in circuit, landing, crosswind) based on the rules of the air. Finally, from an aircraft's initial position and intention the aircraft's position can be projected into the future with a certain level of certainty.

The scenario concerned in this paper is that a UAV is making an arrival into an uncontrolled licensed aerodrome. Unlike a controlled aerodrome where ATC is responsible for all separation between aircraft and their navigation, in an uncontrolled aerodrome the service is completely non authoritative. Therefore, the pilots, or equivalently UAVs, are completely responsible for their own operations. Another challenge associated with uncontrolled aerodromes is that modern Automated Dependant Surveillance - Broadcast (ADS-B) system that can directly transmit aircrafts state may not be available. This means the UAV has to rely solely on its onboard sensors to first detect and track traffic aircraft and then to infer the traffic aircraft's intention. The first part of the problem in general is considered as the sense and avoid capability for UAVs [5, 6]. Many existing research studies in this area focus on the development of sensor systems to detect and track the traffic aircraft the by combing observations from different sensors such the radar [7] and vision sensors [8], where different data fusion algorithms ranging from Kalman filters to particle filters are used [9]. However, most of existing estimation algorithms only utilise the sensor observations and little has been done in terms of inference of aircraft's intention.

To improve the situation awareness of a UAV in uncontrolled aerodrome, the main contribution of paper is to incorporate aerodrome circuit flight rules into the filtering process through probabilistic modelling, since they are able to provide an extra set of information. Therefore, the knowledge of the circuit patten needs to be converted into a probabilistic representation so that it can be exploited by the filtering process. Specifically, two major enabling techniques are adopted in this study. First, multiple models of aircraft dynamics are adopted to characterise the behaviours at different flight phases. To further reflect the flight direction in each circuit phase, minimum velocity constraints are applied on each model by taking into account the stall speed of the aircraft. Second, the transition behaviours between consecutive flight phases are described in a state-dependent and stochastic manner. Compared with the traditional Markov jump transition techniques (see, e.g. ref [10]), the state-dependent transition (SDT) allows a more precise description of a phase transition mechanism, whereas the stochastic modelling is able to capture the uncertainties during this process.

It is a challenging task to solve the corresponding multi-mode target tracking problem after incorporating the circuit knowledge due to the nonlinear/non-Gaussian nature of this kind of problems. If both the target model and sensor model are linear and the transition behaviour can be described as a Markov jump process, a popular solution is to use an interacting multiple model (IMM) scheme based on the Kalman filters [10, 11]. In the case where nonlinear system models are involved, IMM particle filter $(\mathrm{PF})$ solutions can be used $[12,13]$. Moreover, when the transition behaviour are modelled as a linear state-dependent function with Gaussian stochastic uncertainties, Seah and Hwang have developed a promising hybrid estimation framework based on multiple Kalman filters under Gaussian assumptions [14, 15]. However, when both the system models and the state-dependent transition are described in 
nonlinear forms, Kalman filter based algorithms are no longer applicable. To this end, this paper aims to develop a new filtering algorithm that is able to deal with nonlinear stochastic transition within the general IMM Bayesian filtering framework [16]. To actually implement the proposed Bayesian filter for the formulated knowledge enhanced target tracking, a particle filter solution is developed in this work, which is able to take into account the state constraints and calculate the transition probabilities.

The remainder of this paper is organised as follows. First, the visual flight rules applied in uncontrolled aerodrome are introduced. Then, by taking into account the knowledge of flight rules, the target tracking problem is formulated as a hybrid state estimation problem. To solve this problem, the next section develops a new IMM Bayesian filtering process, following by the particle filter implementation. To demonstrate the performance of the proposed target tracking algorithm using circuit knowledge, Monte Carlo (MC) simulations are carried out based on a case study scenario. Finally, conclusions of this study are given in the last section.

\section{Visual flight rules in terminal areas}

This section aims to further explain the terminal area environment considered in this study. Essentially, a terminal area is the airspace surrounding an airfield which is mainly used by aircraft arriving at or departing from that airfield [17]. There are effectively two main types of airfield, controlled and uncontrolled. Terminal areas are normally within controlled airspace such as Aerodrome Traffic Zone (ATZ) or a Controlled Traffic Region (CTR) for larger airports. In controlled airfields, ATC has full authority over all aircraft moment with in its zone, which is responsible for all separation between aircraft, and their navigation. Consequently, this means that situation awareness from an individual aircraft is much less important, given that most of the decisions are made by ATC. In contrast, uncontrolled airfields will normally be smaller and instead of an ATC will only have aircraft flight information service (AFIS), air ground radio, or no radio at all. These are non-authoritative services that provide aircraft with information about traffic weather conditions and aerodrome information. At these aerodromes the pilot, or equivalently a UAV, is completely responsible for their own operations and no clearances are required, which means a high level of situation awareness is needed. Therefore, this paper mainly consider the aerodrome in the latter case.

Given no ATC service available, aircraft landing at an uncontrolled aerodrome are required to follow the Visual Flight Rule (VFR), which consists of a number of standard procedures. The circuit flight pattern is the most significant VFR procedure. The circuit is a rectangular flight path flown by all arriving aircraft which ensures that the runway and all other traffic remain in sight at all times. There are four distinct phases of the circuit, namely crosswind leg, downwind leg, base leg and final, which are flown in that order. A typical procedure for an aircraft to join an airfield traffic circuit is the standard Over Head Joint (OHJ). An OHJ is performed by first flying overhead to observe the conditions (wind sock and runway surface) and any traffic in the circuit. Once an over flight has been conducted, a descent to circuit height (usually 1000ft Above Aerodrome Level (AAL)) is made and the aircraft joins the circuit via the crosswind leg. However, aircraft are not legally required to perform this particular approach, they may join at any point on the circuit. This is some times done to shorten the approach to save on fuel and flight time. However, as this is not the norm, the joining aircraft needs to have enough information to prevent the conflict with other traffic already in the circuit. 


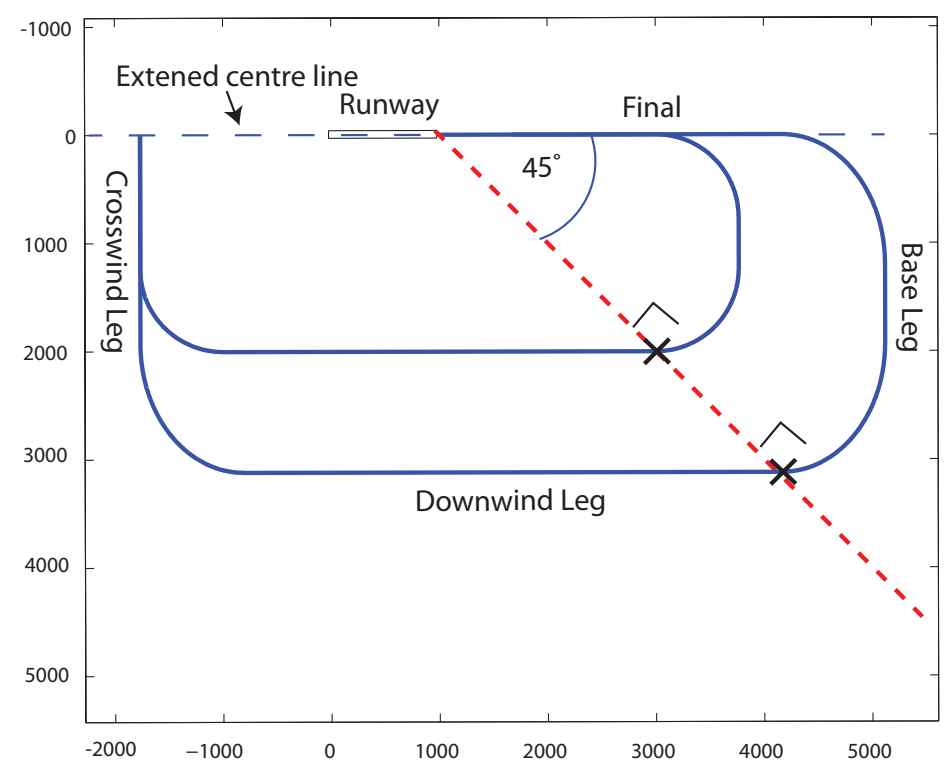

Figure 1: Circuit dimensions

The exact dimensions of a circuit are not completely fixed but can be greatly constrained by a couple of rules. If an aircraft were to join on the crosswind leg they would approach it from the dead side (non traffic side) descending to circuit height before joining. At an appropriate distance from the runway the aircraft would turn either right or left by $90^{\circ}$ depending on the circuit direction onto the downwind leg. This is normally done using a steady rate one turn, which is about $6 \mathrm{deg} / \mathrm{s}$. The downwind leg is flown parallel to the runway, until the aircraft is at a relative bearing of $45^{\circ}$ from the threshold point of the runway from the extended centreline [18], where another $90^{\circ}$ turn is made to the base leg. Somewhere around the beginning of the base leg the aircraft will start descending, and slowing until the aircraft reaches $V_{F E}$ the speed where it can extend its flaps. Finally a turn on to the final leg to land is made. The aircraft will aim to be at a height of no less than $500 \mathrm{ft}$ at the end of the turn to final. This turn is make so that by the end of the turn the aircraft is flying straight at the runway down the extended centreline. More detailed guidance to circuit pattens under Visual Flight Rules (VFR) can be found in ref $[19,20]$.

As explained in the previous rectangular nature of the circuit, relating to the runway makes it easy to define the circuit pattern. Hence, to facilitate the modelling of the circuit flight rule in a terminal area, a local coordinate is defined with respect to the active runway and the current wind direction (see Fig. 1). The origin of the local coordinate is located at the runway threshold point, the $x$ axis is aligned with the runway centreline towards the downwind direction, and the $y$ axis is aligned with the base leg direction.

For a UAV intending to land at an uncontrolled airfield, it needs to be aware of other aircraft within this terminal region, especially those who are already in the landing circuit and may breach the separation criterion in the near future. In order to coordinate with other aircraft to guarantee the self-separation, it is essential to acquire the status of traffic aircraft and their intentions, i.e. the circuit phases. Generally, this problem can be cast into a target tracking problem based on the understanding of the target's motion and the observation information from sensors. However, in the uncontrolled aerodrome the circuit flight pattern 
can also provide extra knowledge about the aircraft's behaviour, which should be exploited in the filter process.

\section{Problem formulation}

The target tracking problem of using UAVs' radar sensors and general circuit flight knowledge is formulated in this section. First, the aircraft's dynamics associated with each fight phase are modelled. Then, the phase transition behaviours are described in a novel state-dependent stochastic form. Finally, in conjunction with the sensor model, the estimation problem is formulated in a probabilistic framework.

\subsection{Aircraft model}

To estimate the state of a traffic aircraft in the circuit, a mathematical model is required to describe its dynamical behaviour. Although in general the aircraft's state can be described by the position and velocity, the aircraft may possess different dynamical features in different circuit phases. To more precisely describe the aircraft motions along the circuit, multiple models are adopted which represent different circuit phases correspondingly. Since the circuit height is usually pre-determined (e.g. 1000ft) [18] and collisions are more likely to occur when aircraft join the circuit at the same height, this paper only focuses on the horizontal motions of the aircraft in 2-dimensions. Given the aircraft position $(x, y)$ in local coordinates, the aircraft state at time index $k$ can be defined as $\mathbf{x}_{k}=\left[\begin{array}{llll}x_{k} & \dot{x}_{k} & y_{k} & \dot{y}_{k}\end{array}\right]^{T}$, which also includes the velocity. A number of candidate models are summarised as follows.

\subsubsection{Constant velocity model}

The aircraft flying in the crosswind and downwind leg can be described by the constant velocity $(\mathrm{CV})$ model, such that

$$
\mathbf{x}_{k+1}=\left[\begin{array}{cccc}
1 & T & 0 & 0 \\
0 & 1 & 0 & 0 \\
0 & 0 & 1 & T \\
0 & 0 & 0 & 1
\end{array}\right] \mathbf{x}_{k}+\left[\begin{array}{cc}
\frac{T^{2}}{2} & 0 \\
T & 0 \\
0 & \frac{T^{2}}{2} \\
0 & T
\end{array}\right] \mathbf{w}_{k},
$$

where $k$ is the time index, $T$ is the sampling time, $\mathbf{x}_{k} \in \mathbb{R}^{4}$ is the aircraft state, and $\mathbf{w}_{k} \in \mathbb{R}^{2}$ is the process noise. The noise vector $\mathbf{w}_{k}$ is assumed to be Gaussian with zero mean vector and covariance matrix $Q_{k}$.

\subsubsection{White-noise acceleration model}

The aircraft flying in base leg and final approach are more likely to reduce their speed rather than maintaining a constant cruise. The simplest model for describing this motion is a whitenoise acceleration (WA) model, which differs from the CV model (1) only in the noise level $[21]$. 


\subsubsection{Coordinated turn model}

The model associated with the turning phases, including turning onto the downwind leg, base leg and final approach, can be described by the coordinated turn (CT) model

$$
\begin{aligned}
\mathbf{x}_{k+1}= & {\left[\begin{array}{cccc}
1 & \frac{\sin (\omega T)}{\omega} & 0 & -\frac{1-\cos (\omega T)}{\omega} \\
0 & \cos (\omega T) & 0 & -\sin (\omega T) \\
0 & \frac{1-\cos (\omega T)}{\omega} & 1 & \frac{\sin (\omega T)}{\omega} \\
0 & \sin (\omega T) & 0 & \cos (\omega T)
\end{array}\right] \mathbf{x}_{k} } \\
& +\left[\begin{array}{cc}
\frac{T^{2}}{2} & 0 \\
T & 0 \\
0 & \frac{T^{2}}{2} \\
0 & T
\end{array}\right] \mathbf{w}_{k},
\end{aligned}
$$

where $\omega$ is the nominal turning rate and the Gaussian process noise $\mathbf{w}_{k}$ is used to capture the uncertainties.

In addition to the multiple models which are used to distinguish between the flight phases, other information on circuit flight can also be used in the target tracking algorithm to improve the situation awareness.

\subsection{Discrete mode transition}

To incorporate the multiple models associated with the circuit flight, a discrete mode variable $m_{k} \in \mathcal{M}$ is introduced to indicate the current phase of the aircraft, where $\mathcal{M}=\{1,2, \ldots, M\}$ is the set of $M$ circuit phases. From the crosswind to final approach, the circuit phases and the corresponding model are summarised in Table 1.

Table 1: Circuit phases and aircraft models.

\begin{tabular}{c|l|c}
\hline Mode & Circuit phase & Aircraft Model \\
\hline 1 & crosswind & $\mathrm{CV}$ \\
2 & turning into downwind & $\mathrm{CT}$ \\
3 & downwind & $\mathrm{CV}$ \\
4 & turning into base & $\mathrm{CT}$ \\
5 & base & WA \\
6 & turning into final & $\mathrm{CT}$ \\
7 & final & WA \\
\hline
\end{tabular}

The mode transition process can be described in a probabilistic way. Suppose at time $k$ the transition probability from mode $i$ to mode $j$ is denoted as $\pi_{i j}\left(\mathbf{x}_{k}\right)$, where $i, j \in \mathcal{M}$. A commonly used method for modelling this process assumes the Markov jump model where the probability $\pi_{i j}$ is assumed to be a known constant and independent of the state $\mathbf{x}_{k}$. Although this method is very popular in the target tracking field, it cannot fully take into account the flight patterns in the circuit. As mentioned earlier there is no nominal circuit path for all aircraft, the pilots usually execute the circuit flight by following a number of rules. Therefore, it is necessary to develop a dedicated transition model for the circuit flight.

In this paper, the stochastic transition criterion from mode $i$ to mode $j$ are modelled as a nonlinear function of state $\mathbf{x}_{k}$ in conjunction with a random vector to capture the 
uncertainties, such that

$$
\mathbf{G}_{i j}\left(\mathbf{x}_{k}\right)+\gamma_{i j} \leq 0
$$

where $G_{i j}\left(\mathbf{x}_{k}\right)$ is a general nonlinear function of $\mathbf{x}_{k}$ and $\gamma_{i j}$ is a random vector satisfying a distribution $p_{i j}(\gamma)$. Thus, the mode transition probability can be expressed as

$$
\pi_{i j}\left(\mathbf{x}_{k}\right)=\operatorname{Pr}\left\{G_{i j}\left(\mathbf{x}_{k}\right)+\gamma_{i j} \leq 0\right\}
$$

In particular, when $\gamma_{i j}$ follows a Gaussian distribution with zero mean and standard deviation of $\sigma,(4)$ can be written as

$$
\pi_{i j}\left(\mathbf{x}_{k}\right)=\Phi\left(-G_{i j}\left(\mathbf{x}_{k}\right) ; 0, \sigma^{2}\right)=1-\Phi\left(G_{i j}\left(\mathbf{x}_{k}\right) ; 0, \sigma^{2}\right),
$$

where $\Phi\left(y ; 0, \sigma^{2}\right)=\int_{\infty}^{y} \mathcal{N}\left(u ; 0, \sigma^{2}\right) \mathrm{d} u$ denotes the cumulative distribution function (CDF) with zero mean and standard deviation $\sigma$. Note that the constructed mode transition probabilities need to be normalised such that $\sum_{j \in \mathcal{M}} \pi_{i j}\left(\mathbf{x}_{k}\right)=1$.

The state-dependent transition has been investigated in some pioneer works. Blom and Bloem have provided an example where the transition probability $\pi_{i j}\left(\mathbf{x}_{k}\right)$ is a piecewise linear function of state $\mathbf{x}_{k}$ [16]. Multiple Markov jump probabilities that depend on the state are used in ref [22] to distinguish transition behaviours associated with different system modes. A more detailed modelling of using random variables to capture the transition uncertainties has been shown in a series of hybrid state estimation algorithms [14, 15], where the statedependent function and the integration of the random variables have to follow a linear form. Comparing to existing work, this paper adopts a general nonlinear state-dependent function, hence it is able to provide more flexibilities in modelling mode transition behaviours with uncertainties.

\subsection{Hybrid state estimation}

Combining the multiple models described in Eq. (1)-(2) and the transition of the discrete mode variable $m_{k}$, the motions and intentions of the traffic aircraft in the circuit can be cast into a hybrid system

$$
\mathbf{x}_{k+1}=\mathbf{f}\left(\mathbf{x}_{k}, m_{k}, \mathbf{w}_{k}\right), \quad m_{k} \in \mathcal{M}
$$

where the discrete state $m_{k}$ determines the aircraft model and the associated process noise $\mathbf{w}_{k}$ that govern the evolution of continuous state $\mathbf{x}_{k}$.

In order to obtain a greater situation awareness about the traffic aircraft described in Eq. (6) without any direct measurements from transponders, a UAV needs to rely on its onboard sensors to infer the states and intentions of traffic aircraft. In this paper, a radar system is considered, which can be modelled as a range and bearing function such that

$$
\mathbf{z}_{k}=\left[\begin{array}{c}
\sqrt{\left(x_{k}-\bar{x}_{k}\right)^{2}+\left(y_{k}-\bar{y}_{k}\right)^{2}} \\
\arctan \left(\frac{y_{k}-\bar{y}_{k}}{x_{k}-\bar{x}_{k}}\right)
\end{array}\right]+\mathbf{v}_{k}
$$

where $\left(\bar{x}_{k}, \bar{y}_{k}\right)$ denotes the current UAV position, $\mathbf{z}_{k}=\left[\begin{array}{ll}r_{k} & \theta_{k}\end{array}\right]^{T}$ denotes the observation vector including the range $r_{k}$ and the bearing angle $\theta_{k}$ between the UAV and the target aircraft and $\mathbf{v}_{k}$ denotes the observation noise vector which is Gaussian with zero mean vector and covariance matrix $R$. 
The current situation of the traffic aircraft can be represented by the probabilistic distribution of the aircraft's state and the associated mode. Therefore, the awareness of the aircraft involves the estimate of the distribution $p\left(\mathbf{x}_{k}, m_{k} \mid \mathbf{z}_{1: k}\right)$ at time $k$ based on the observation sequence $\mathbf{z}_{1: k}=\left\{\mathbf{z}_{1}, \ldots, \mathbf{z}_{k}\right\}$ and the initial distribution $p\left(\mathbf{x}_{0}, m_{0}\right)$, which in turn can be done in a recursive probabilistic inference process.

\section{Bayesian filtering}

Bayesian filtering provides a generic mathematical tool for probabilistic inference. To deal with the formulated hybrid system, a new IMM filtering scheme is developed in this paper by following the general IMM framework [16]. In particular, it takes into account the probabilistic modelling of mode transition process by calculating the probabilities of mode transition based on the current system state. To implement this nonlinear and non-Gaussian filtering scheme, the sequential Monte Carlo algorithm is used which is also known as the particle filter.

\subsection{IMM Bayesian framework}

The recursive equations for calculating the joint conditional probability density $p\left(\mathbf{x}_{k}, m_{k} \mid \mathbf{z}_{1: k}\right)$ is summarised in this subsection. The detailed derivation can be found in ref [16]. Baye's theorem can be used to construct this conditional density into two steps:

1. State evolution, where the joint prior distribution $p\left(\mathbf{x}_{k}, m_{k} \mid \mathbf{z}_{1: k-1}\right)$ is obtained from the distribution $p\left(\mathbf{x}_{k-1}, m_{k-1} \mid \mathbf{z}_{1: k-1}\right)$ in the previous time step.

2. Bayes measurement update, where the prior distribution is corrected by the likelihood function to produce the posterior distribution

$$
p\left(\mathbf{x}_{k}, m_{k} \mid \mathbf{z}_{1: k}\right)=\frac{p\left(\mathbf{z}_{k} \mid \mathbf{x}_{k}, m_{k}\right) p\left(\mathbf{x}_{k}, m_{k} \mid \mathbf{z}_{1: k-1}\right)}{p\left(\mathbf{z}_{k} \mid \mathbf{z}_{1: k-1}\right)}
$$

By the law of total probability, the state evolution step can be further detailed as

$$
\begin{aligned}
& p\left(\mathbf{x}_{k}, m_{k}^{j} \mid \mathbf{z}_{1: k-1}\right) \\
& =\int_{\mathbb{R}^{n}} p\left(\mathbf{x}_{k} \mid \mathbf{x}_{k-1}, m_{k}^{j}, \mathbf{z}_{1: k-1}\right) p\left(\mathbf{x}_{k-1}, m_{k}^{j} \mid \mathbf{z}_{1: k-1}\right) \mathrm{d} \mathbf{x}_{k-1}
\end{aligned}
$$

where $m_{k}^{j}$ denotes that the discrete mode $m_{k}$ takes the value of $j$.

Applying Chapman-Kolmogorov equation to the second distribution in Eq. (9) yields

$$
\begin{aligned}
& p\left(\mathbf{x}_{k-1}, m_{k}^{j} \mid \mathbf{z}_{1: k-1}\right) \\
& =\sum_{i \in \mathbb{M}} p\left(m_{k}^{j} \mid m_{k-1}^{i}, \mathbf{x}_{k-1}\right) p\left(\mathbf{x}_{k-1}, m_{k-1}^{i} \mid \mathbf{z}_{1: k-1}\right),
\end{aligned}
$$

where $m_{k-1}^{i}$ denotes that $m_{k-1}=i$. It can be noted that the state-dependent transition probability in Eq. (10) can be denoted as

$$
\Pi_{i j}\left(\mathbf{x}_{k-1}\right)=p\left(m_{k}^{j} \mid m_{k-1}^{i}, \mathbf{x}_{k-1}\right), \quad \forall i, j \in \mathcal{M}
$$


Combining Eqs. (8)-(11), the Bayesian inference for the joint conditional distribution can be summarised as

$$
\begin{aligned}
p & \left(\mathbf{x}_{k}, m_{k}^{j} \mid \mathbf{z}_{1: k}\right) \\
= & \frac{1}{c_{t}} p\left(\mathbf{z}_{k} \mid \mathbf{x}_{k}, m_{k}^{j}\right) \int_{\mathbb{R}^{n}} p\left(\mathbf{x}_{k} \mid \mathbf{x}_{k-1}, m_{k}^{j}\right) \\
& \sum_{i \in \mathbb{M}} \Pi_{i j}\left(\mathbf{x}_{k-1}\right) p\left(\mathbf{x}_{k-1}, m_{k-1}^{i} \mid \mathbf{z}_{1: k-1}\right) \mathrm{d} \mathbf{x}_{k-1}
\end{aligned}
$$

where $c_{t}$ is the normalisation factor.

To implement the above recursive equation by using a bank of mode-matched filters, the inference process is decomposed into four steps.

\subsubsection{Mode transition}

The evolution of the conditional model probability from $k-1$ to $k$ is characterised as the mode transition step. Using the law of total probability yields

$$
p\left(m_{k}^{j} \mid \mathbf{z}_{1: k-1}\right)=\int_{\mathbb{R}^{n}} p\left(\mathbf{x}_{k-1}, m_{k}^{j} \mid \mathbf{z}_{1: k-1}\right) \mathrm{d} \mathbf{x}_{k-1} .
$$

Substituting Eq. (10) and the subsequent derivations into the above equation gives

$$
\begin{aligned}
p & \left(m_{k}^{j} \mid \mathbf{z}_{1: k-1}\right) \\
= & \int_{\mathbb{R}^{n}} \sum_{i \in \mathbb{M}} \Pi_{i j}\left(\mathbf{x}_{k-1}\right) p\left(\mathbf{x}_{k-1}, m_{k-1}^{i} \mid \mathbf{z}_{1: k-1}\right) \mathrm{d} \mathbf{x}_{k-1} \\
= & \sum_{i \in \mathbb{M}} \int_{\mathbb{R}^{n}} \Pi_{i j}\left(\mathbf{x}_{k-1}\right) p\left(\mathbf{x}_{k-1} \mid m_{k-1}^{i}, \mathbf{z}_{1: k-1}\right) \mathrm{d} \mathbf{x}_{k-1} \\
& p\left(m_{k-1}^{i} \mid \mathbf{z}_{1: k-1}\right) .
\end{aligned}
$$

This relation shows that the predicted model probability $p\left(m_{k}^{j} \mid \mathbf{z}_{1: k-1}\right)$ can be derived from the mode conditional density $p\left(\mathbf{x}_{k-1} \mid m_{k-1}^{i}, \mathbf{z}_{1: k-1}\right)$ and the mode probability $p\left(m_{k-1}^{i} \mid \mathbf{z}_{1: k-1}\right)$.

\subsubsection{State interaction}

The initial state density $p\left(\mathbf{x}_{k-1} \mid m_{k}^{j}, \mathbf{z}_{1: k}\right)$ for each mode-matched filter is calculated in this step by considering the influence of the predicted model probability. Dividing Eq. (10) by $p\left(m_{k}^{j} \mid \mathbf{z}_{1: k-1}\right)$ gives

$$
p\left(\mathbf{x}_{k-1} \mid m_{k}^{j}, \mathbf{z}_{1: k}\right)=\frac{\sum_{i \in \mathbb{M}} \Pi_{i j}\left(\mathbf{x}_{k-1}\right) p\left(\mathbf{x}_{k-1}, m_{k-1}^{i} \mid \mathbf{z}_{1: k-1}\right)}{p\left(m_{k}^{j} \mid \mathbf{z}_{1: k-1}\right)}
$$

\subsubsection{State evolution}

Given the initial state for each mode-matched filter in Eq. (15), the state evolution step propagates these densities from $k-1$ to $k$ through integration, such that

$$
\begin{aligned}
& p\left(\mathbf{x}_{k} \mid m_{k}^{j}, \mathbf{z}_{1: k-1}\right) \\
& =\int_{\mathbb{R}^{n}} p\left(\mathbf{x}_{k} \mid \mathbf{x}_{k-1}, m_{k}^{j}\right) p\left(\mathbf{x}_{k-1} \mid m_{k}^{j}, \mathbf{z}_{1: k-1}\right) \mathrm{d} \mathbf{x}_{k-1}
\end{aligned}
$$


where the transition density $p\left(\mathbf{x}_{k} \mid \mathbf{x}_{k-1}, m_{k}^{j}\right)$ can be obtained from the corresponding models and distributions of process noises.

\subsubsection{Correction}

In this step, the latest measurement information is used to update the prior distribution in each mode-matched filter. Following Eq. (8) gives

$$
\begin{aligned}
& p\left(\mathbf{x}_{k}, m_{k}^{j} \mid \mathbf{z}_{1: k}\right) \\
& \propto p\left(\mathbf{z}_{k} \mid \mathbf{x}_{k}, m_{k}^{j}\right) p\left(\mathbf{x}_{k}, m_{k}^{j} \mid \mathbf{z}_{1: k-1}\right) \\
& \propto p\left(\mathbf{z}_{k} \mid \mathbf{x}_{k}, m_{k}^{j}\right) p\left(\mathbf{x}_{k} \mid m_{k}^{j}, \mathbf{z}_{1: k-1}\right) p\left(m_{k}^{j} \mid \mathbf{z}_{1: k-1}\right)
\end{aligned}
$$

where the likelihood density $p\left(\mathbf{z}_{k} \mid \mathbf{x}_{k}, m_{k}^{j}\right)$ can be obtained from the corresponding observation model and its noise distribution.

To complete the recursive cycle, the required mode-conditioned state distribution and mode probability at the next time step can also be calculated such that

$$
p\left(m_{k}^{j} \mid \mathbf{z}_{1: k}\right)=\int_{\mathbb{R}^{n}} p\left(\mathbf{x}_{k}, m_{k}^{j} \mid \mathbf{z}_{1: k}\right) \mathrm{d} \mathbf{x}_{k}
$$

and

$$
p\left(\mathbf{x}_{k} \mid m_{k}^{j}, \mathbf{z}_{1: k}\right) \propto p\left(\mathbf{z}_{k} \mid \mathbf{x}_{k}, m_{k}^{j}\right) P\left(\mathbf{x}_{k} \mid m_{k}^{j}, \mathbf{z}_{1: k-1}\right) .
$$

\subsection{Particle filter implementation}

The Bayesian filtering scheme provides a general framework for state estimation of the hybrid systems. However, there is no analytical solution due to the nonlinear and non-Gaussian nature associated with the hybrid systems.

Suppose that at time $k-1$ there exists a set of weighted particles $\left\{\mathbf{x}_{k-1}^{i, n}, \mu_{k-1}^{i, n} ; i \in \mathcal{M}, n \in\right.$ $\{1, \ldots, N\}\}$, which spans over the joint distribution

$$
p\left(\mathbf{x}_{k-1}, m_{k-1}^{i} \mid Z^{k-1}\right) \approx \sum_{n=1}^{N} \mu_{k-1}^{i, k} \delta\left(\mathbf{x}_{k-1}-\mathbf{x}_{k-1}^{i, k}\right)
$$

where $\delta(\cdot)$ is a Dirac delta function. For each discrete mode $i \in \mathcal{M}, N$ particles are allocated to the corresponding mode-matched filter with a total number of $N_{p}=N \cdot M$ weighted particles.

The development of Sequential Monte Carlo implementation involves the substitution of the empirical density Eq. (20) into four filtering cycles, respectively. Firstly, the prior mode probability in Eq. (14) is approximated as

$$
p\left(m_{k}^{j} \mid \mathbf{z}_{1: k-1}\right) \approx \sum_{i=1}^{M} \sum_{n=1}^{N} \pi_{i j}\left(\mathbf{x}_{k-1}^{i, n}\right) \cdot \mu_{k-1}^{i, n} \triangleq \Lambda_{k-1}^{j}
$$

where the notation $\Lambda_{k-1}^{j}$ is defined to facilitate the rest of derivation. 
Secondly, the initial density for each mode-conditioned filter can be approximated by inserting the particles into Eq. (15) such that

$$
\begin{aligned}
& p\left(\mathbf{x}_{k-1} \mid m_{k}^{j}, \mathbf{z}_{1: k-1}\right) \\
& \approx \sum_{i=1}^{M} \sum_{n=1}^{N} \pi_{i j}\left(\mathbf{x}_{k-1}^{i, n}\right) \mu_{k-1}^{i, n} \delta\left(\mathbf{x}_{k-1}-\mathbf{x}_{k-1}^{i, n}\right) / \Lambda_{k-1}^{j}
\end{aligned}
$$

where the density $p\left(\mathbf{x}_{k-1} \mid m_{k}^{j}, \mathbf{z}_{1: k-1}\right)$ is approximated by $N_{p}$ particles instead of $N$. A solution to this problem as suggested in ref [16] is to perform a resampling process so that $N$ samples $\left\{\overline{\mathbf{x}}_{k-1}^{j, n}, \bar{\mu}_{k-1}^{j, n}\right\} \sim p\left(\mathbf{x}_{k-1} \mid m_{k}^{j}, \mathbf{z}_{1: k-1}\right)$ are generated for each mode.

The third step is to propagate the mode-conditioned density derived in (22) to the next time step $k$. Following (16), new samples $\left\{\mathbf{x}_{k}^{j, n}\right\}$ that are draw from $p\left(\mathbf{x}_{k} \mid \mathbf{x}_{k-1}, m_{k}^{j}\right)$ given the resampled particles $\left\{\overline{\mathbf{x}}_{k-1}^{j, n}, \bar{\mu}_{k-1}^{j, n}\right\}$ can be used to approximate the prior density

$$
p\left(\mathbf{x}_{k} \mid \mathbf{x}_{k-1}, m_{k}^{j}\right) \approx \sum_{n=1}^{N} \bar{\mu}_{k}^{j, n} \delta\left(\mathbf{x}_{k}-\overline{\mathbf{x}}_{k}^{j, n}\right) .
$$

The last step in the filtering cycle aims to update the weights using the likelihood function. Inserting Eq. (23) and the predicted mode probability $\Lambda_{k-1}^{j}$ into Eq. (17) gives

$$
\begin{aligned}
& p\left(\mathbf{x}_{k}, m_{k}^{j} \mid \mathbf{z}_{1: k}\right) \\
& \propto \sum_{n=1}^{N} p\left(\mathbf{z}_{k} \mid \mathbf{x}_{k}^{j, n}, m_{k}^{j}\right) \bar{\mu}_{k}^{j, n} \delta\left(\mathbf{x}_{k}-\mathbf{x}_{k}^{j, n}\right) \Lambda_{k-1}^{j} .
\end{aligned}
$$

Thus, the samples remain the same, whereas the new weights can be updated as

$$
\mu_{k}^{j, n}=\frac{p\left(\mathbf{z}_{k} \mid \mathbf{x}_{k}^{j, n}, m_{k}^{j}\right) \Lambda_{k-1}^{j} \bar{\mu}_{k}^{j, n}}{\sum_{j=1}^{M} \sum_{n=1}^{N} \bar{\mu}_{k}^{j, n}} .
$$

\section{Case study}

A case study is designed to demonstrate the performance of the proposed target tracking algorithm within a terminal region where the visual flight rules apply. Specifically, it is concerned with the arrival and landing of a UAV to a licensed uncontrolled aerodrome, e.g. Wellsbourne aerodrome in UK. In order to speed up its arrival the UAV is assumed to request a straight in approach. However, this means it has to deal with other traffic aircraft and use information from the onboard radar and knowledge of arrival and circuit procedures to gain enough situation awareness to make a safe arrival. More importantly, the UAV needs to give way to any other aircraft already established and flying in the circuit pattern, which in turn requires at least awareness of the location, speed and circuit phase of the traffic aircraft.

This example scenario is illustrated in Fig 2, where three different circuit phases, namely downwind (Phase 3), turning into base (Phase 4) and the base leg (Phase 5), are considered during the simulation. It is assumed that at time $k=0$ a traffic aircraft is located in the downwind phase with the coordinates $(1000 \mathrm{~m},-2000 \mathrm{~m})$ and speed of $50 \mathrm{~m} / \mathrm{s}$ when it is reliably detected by the UAV radar. It follows the nominal circuit flight rules, i.e. with constant speed 
and $0^{\circ}$ heading angle in downwind and then turns onto base at the standard changing point at $(-2000 \mathrm{~m},-2000 \mathrm{~m})$ with a turning rate about $6^{\circ} / \mathrm{s}$. Once established on the base leg indicated by the $90^{\circ}$ heading angle, it starts to decrease its speed at an acceleration of $-1 \mathrm{~m} / \mathrm{s}^{2}$. On the other hand, the UAV is assumed to fly toward the runway in a straight-line at the speed of $40 \mathrm{~m} / \mathrm{s}$ starting from the coordinate $(6000 \mathrm{~m}, 0)$.

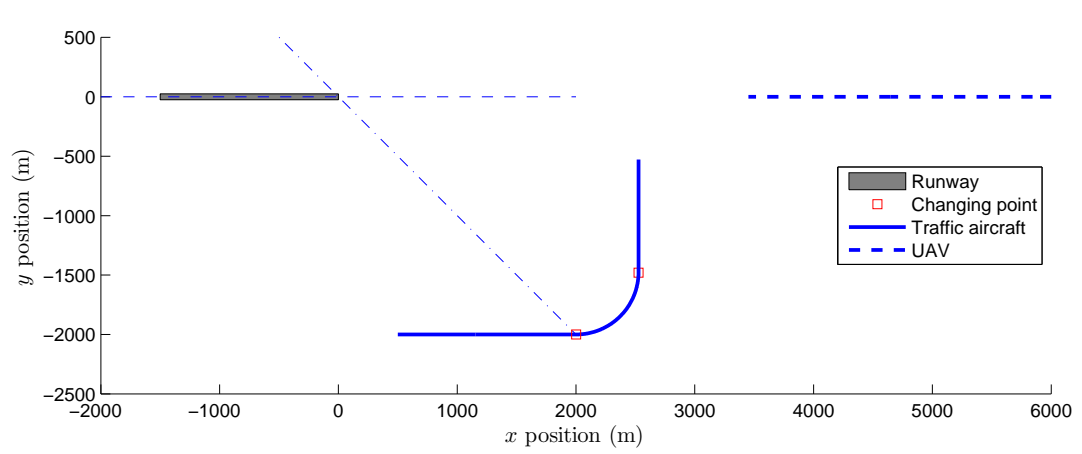

Figure 2: Target tracking scenario

Following the general rules of air, the nominal transition criteria for turning into base can be described by the $45^{\circ}$ line which can be written as $\mathcal{C}_{34}=x_{k}+y_{k} \geq 0$. Since a human pilot may not be able to fly the circuit perfectly, a random variable $\gamma_{34}$ is introduced to characterise the stochastic transition behaviour. Thus, the state-dependent transition criteria can be modelled as $\mathcal{C}_{34}=\left\{x_{k}+y_{k} \geq \gamma\right\}$. In this paper the random variable is assumed to follow a Gaussian distribution with a zero mean, i.e. $\gamma_{34} \sim \mathcal{N}\left(\gamma ; 0, \sigma_{34}^{2}\right)$ which is a common engineering assumption. The standard deviation in the simulation is chosen to be $\sigma_{1}=200 \mathrm{~m}$ to capture the uncertainty in making the turning decision. The associated state-dependent transition probability can be evaluated as $\pi_{34}\left(\mathbf{x}_{k}\right)=\operatorname{Pr}\left\{\mathcal{C}_{34} \mid \mathbf{x}_{k}\right\}=\Phi\left(\mathcal{C}_{34} ; 0, \sigma_{34}^{2}\right)$. Similarly, the transition criteria from mode 4 to mode 5 can be written as $\mathcal{C}_{45}=\arctan \left(\frac{\dot{y}_{k}}{\dot{x}_{k}}\right)-85 \geq+\gamma_{45}$, where the Gaussian random variable $\gamma_{45}$ is zero mean with standard deviation of $5^{\circ}$. To capture any exception in modelling the stochastic transition modelling, a small number of transition probabilities are added into the theoretical ones. Therefore, the state-dependent transition probability matrix $\pi\left(\mathbf{x}_{k}\right) \in \mathbb{R}^{3 \times 3}$ used in this case study can be defined as

$$
\begin{aligned}
& \pi_{11}=\left[1-\Phi\left(\mathcal{C}_{34} ; 0, \sigma_{34}^{2}\right)\right] / \kappa \\
& \pi_{12}=\left[\Phi\left(\mathcal{C}_{34} ; 0, \sigma_{34}^{2}\right)+0.005\right] / \kappa \\
& \pi_{13}=0.005 / \kappa \\
& \pi_{21}=0.005 / \kappa \\
& \pi_{22}=\left[1-\Phi\left(\mathcal{C}_{45} ; 0, \sigma_{34}^{2}\right)\right] / \kappa \\
& \pi_{23}=\left[\Phi\left(\mathcal{C}_{45} ; 0, \sigma_{45}^{2}\right)+0.005\right] / \kappa
\end{aligned}
$$

and for the last row $\pi_{31}=0.005, \pi_{32}=0.005$ and $\pi_{33}=0.99$. The normalisation factor can be calculated to be $\kappa=1.01$.

In the case study, the proposed SDT IMM particle filter is compared with traditional Markov jump based IMM particle filters with constant state transition matrices independent 
of the system state. Two designs of this kind filters (denoted as IMM-PF-1 and IMM-PF-2, respectively) are considered where the the mode transition matrices are

$$
\Pi_{1}=\left[\begin{array}{lll}
0.96 & 0.02 & 0.02 \\
0.02 & 0.96 & 0.02 \\
0.02 & 0.02 & 0.96
\end{array}\right] \Pi_{2}=\left[\begin{array}{ccc}
0.8 & 0.1 & 0.1 \\
0.1 & 0.8 & 0.1 \\
0.1 & 0.1 & 0.8
\end{array}\right]
$$

where $\Pi_{2}$ represents a higher chance of transition than $\Pi_{1}$.

In designing target tracking algorithms, the process noises for different flight modes are set as zero mean Gaussian with different covariance matrices defined as

$$
\begin{aligned}
\sigma_{c v} & =\operatorname{diag}\left\{(1 \mathrm{~m} / \mathrm{s})^{2},(0.5 \mathrm{~m} / \mathrm{s})^{2}\right\}, \\
\sigma_{c t} & =\operatorname{diag}\left\{(1.5 \mathrm{~m} / \mathrm{s})^{2},(1.5 \mathrm{~m} / \mathrm{s})^{2}\right\}
\end{aligned}
$$

and

$$
\sigma_{w a}=\operatorname{diag}\left\{(0.5 \mathrm{~m} / \mathrm{s})^{2},(1.5 \mathrm{~m} / \mathrm{s})^{2}\right\},
$$

respectively. It can be noted that for $\mathrm{CV}$ and WA modes the covariances are directional to reflect the corresponding directions of motion. Moreover, to comply with the stall speed the forward speeds in those models need to satisfy the constraints $\dot{\mathbf{x}}_{k} \geq 20 \mathrm{~m} / \mathrm{s}, \dot{\mathbf{y}}_{k} \geq 20 \mathrm{~m} / \mathrm{s}$, respectively. These state constraints can be incorporated in the particle filter by using the constrained likelihood function[23]. The observation noise $\mathbf{v}_{k}$ introduced in (7) is also assumed to be a Gaussian random variable which has zero mean and covariance matrix $\sigma_{v}=$ $\operatorname{diag}\left\{(20 \mathrm{~m})^{2},\left(1^{\circ}\right)^{2}\right\}$. In this simulation, the initial state distribution for the filters is set to be Gaussian, with mean $\mathbf{x}_{0}=\left[\begin{array}{llll}450 & 50 & -1950 & 0\end{array}\right]^{T}$ and covariance $P_{0}=\operatorname{diag}\{100,10,100,5\}$. The initial mode probabilities are $p\left(m_{0}^{1}\right)=0.7, p\left(m_{0}^{2}\right)=0.3$ and $p\left(m_{0}^{3}\right)=0$. The sampling interval is $T=1$ second and the simulation runs for 50 time steps. The particle number used in the simulation is $N=1000$.

To demonstrate the target tracking performance on the proposed scenario, Monte Carlo simulations were carried out for $L=100$ random generated tracks. Some example trajectories are given Fig 3, where the mode changing points can be varied in a stochastic manner. The algorithm performances in terms of the tracking accuracy were evaluated by Root Mean Square Errors (RMSE) of the position and velocity, respectively. The time histories of RMSE are given in Fig. 4 and Fig. 5. The statistics of the MC simulation results are displayed in Table 2, including average RMSE (aRMSE) for both position and velocity and the mode error count (MEC) of mode estimate. It can be observed that the proposed SDT based IMMPF outperforms the IMM-PFs based on constant transition probabilities in both position and velocity estimation because the circuit knowledge is more effectively incorporated in the filtering process in the proposed method.

Table 2: MC simulation results.

\begin{tabular}{l|c|c|c}
\hline Filters & aRMSE (pos.) & aRMSE (vel.) & MEC \\
\hline SDT-IMM-PF & 23.5 & 4.1 & 2.8 \\
IMM-PF-1 & 25.8 & 6.1 & 13.0 \\
IMM-PF-2 & 29.1 & 8.2 & 14.3 \\
\hline
\end{tabular}

The mode estimation accuracy is also an important measure of the performance. The estimated mode probabilities for a typical simulation run are shown in Fig. 6 It can be seen 


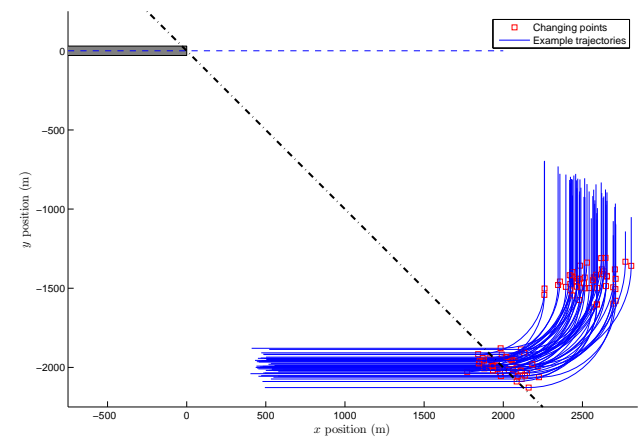

Figure 3: Example trajecotries for the MC simulation

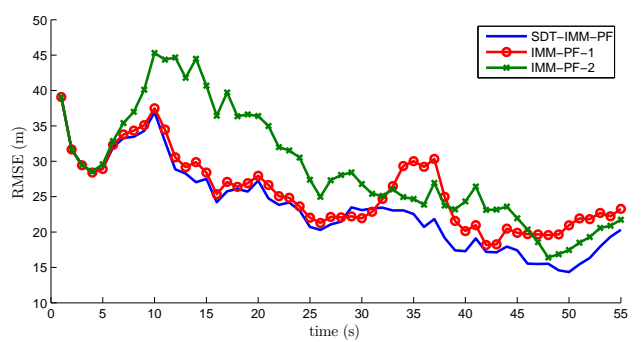

Figure 4: RMSE of position estimation

that the IMM-PF-1 with lower transition probability shows significant delays in estimating the mode transition, whereas the result from IMM-PF-2 has a very susceptible mode estimate, which is especially problematic for steady-state motion. The proposed SDT IMM particle filter on the other hand is able to demonstrate a better mode probability history. The counts of mode estimation errors during the simulation can be found in in Table 2 .

\section{Conclusions}

Inserting a UAV into an uncontrolled aerodrome requires a good level of situation awareness on other traffic aircraft in the same terminal area. In estimating the traffic aircraft's dynamical state and intention, this paper investigates the effectiveness of incorporating the circuit flight

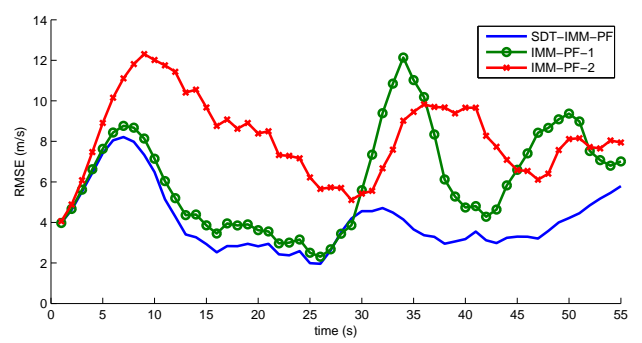

Figure 5: RMSE of velocity estimation 


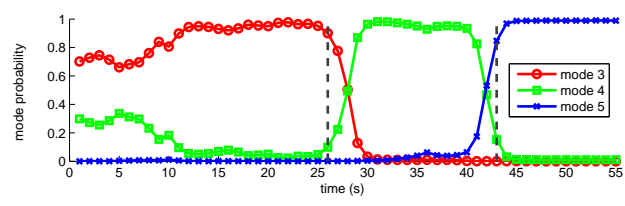

(a) Mode estimation of SDT-IMM-PF

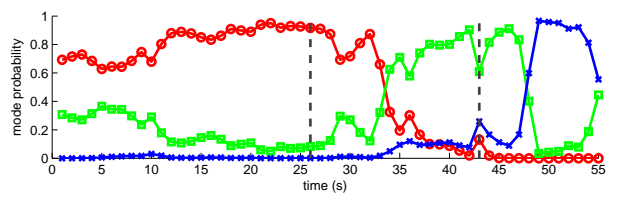

(b) Mode estimation of IMM-PF-1

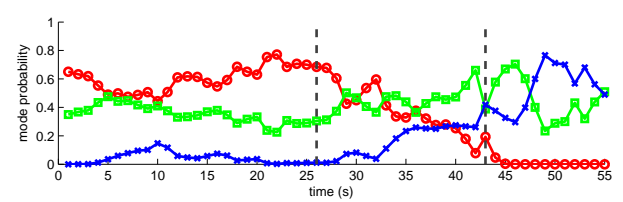

(c) Mode estimation of IMM-PF-2

Figure 6: Mode estimation for a typical simulation run

rules into the traditional sensor based target tracking. In formulating this target tracking problem, multiple models are used to represent the different motion behaviours in different fight phases and the stochastic state-dependent transitions are used to characterise the actual phase transition with uncertainties. The resulted hybrid estimation problem is solved by the proposed SDT IMM particle filter, where the mode transition probabilities is calculated as a cumulative density function of system state instead of using a constant matrix. This algorithm was compared with the general Markov jump based IMM PFs in the case study scenario. The MC simulations show that the proposed algorithm is not only able to obtain a better position and velocity accuracy but also a significantly improved mode estimate, which can more clearly indicate the intentions of the traffic aircraft. With the enhanced estimation accuracy on both the dynamical state and intention, the uncertainties in predicting the aircraft's state in the future can also be reduced, which will lead to an improved level of situation awareness for UAVs in the terminal area. The future work in this area will investigate how to exploit circuit information to project the traffic aircraft's motion into future so that autonomous decisions can be made to guarantee the self-separation for UAVs.

\section{Acknowledgements}

This work was supported by the U.K. Engineering and Physical Sciences Research Council (EPSRC) Autonomous and Intelligent Systems programme under the grant number EP/J011525/1 with BAE Systems as the leading industrial partner.

\section{References}

[1] FAA. Integration of civil unmanned aircraft systems (uas) in the national airspace system (nas) roadmap. Technical report, Federal Aviation Administration, 2013.

[2] CAA. Cap 722 unmanned aircraft system operations in uk airspace. Technical report, Civil Aviation Authority, 2012. 
[3] Endsley MR. Design and evaluation for situation awareness enhancement. In Proceedings of the Human Factors and Ergonomics Society Annual Meeting, volume 32. SAGE Publications, pp. 97-101.

[4] McAree O and Chen WH. Artificial situation awareness for increased autonomy of unmanned aerial systems in the terminal area. Journal of Intelligent 83 Robotic Systems 2013; 70(1-4): 545-555. DOI:10.1007/s10846-012-9738-x.

[5] Angelov P. Sense and Avoid in UAS: Research and Applications. Aerospace Series, Wiley, 2012. ISBN 9780470979754.

[6] Prats X, Delgado L, Ramirez J et al. Requirements, issues, and challenges for sense and avoid in unmanned aircraft systems. Journal of aircraft 2012; 49(3): 677-687.

[7] Accardo D, Fasano G, Forlenza L et al. Flight test of a radar-based tracking system for uas sense and avoid. Aerospace and Electronic Systems, IEEE Transactions on 2013; 49(2): 1139-1160. DOI:10.1109/TAES.2013.6494404.

[8] Mcfadyen A and Mejias L. A survey of autonomous vision-based see and avoid for unmanned aircraft systems. Progress in Aerospace Sciences 2015; DOI:doi:10.1016/j. paerosci.2015.10.002. In Press.

[9] Cappello F, Ramasamy S and Sabatini R. Multi-sensor data fusion techniques for rpas detect, track and avoid. In SAE 2015 AeroTech Congress and Exhibition. SAE Technical Paper 2015-01-2475. DOI:10.4271/2015-01-2475.

[10] Mazor E, Averbuch A, Bar-Shalom Y et al. Interacting multiple model methods in target tracking: a survey. Aerospace and Electronic Systems, IEEE Transactions on 1998; 34(1): 103-123. DOI:10.1109/7.640267.

[11] Bar-Shalom Y, Challa S and Blom H. IMM estimator versus optimal estimator for hybrid systems. Aerospace and Electronic Systems, IEEE Transactions on 2005; 41(3): 986-991. DOI:10.1109/TAES.2005.1541443.

[12] Arulampalam M, Gordon N, Orton M et al. A variable structure multiple model particle filter for gmti tracking. In Information Fusion, 2002. Proceedings of the Fifth International Conference on, volume 2. pp. 927-934 vol.2. DOI:10.1109/ICIF.2002.1020911.

[13] Boers Y and Driessen J. Interacting multiple model particle filter. Radar, Sonar and Navigation, IEE Proceedings - 2003; 150(5): 344-349. DOI:10.1049/ip-rsn:20030741.

[14] Seah C and Hwang I. State estimation for stochastic linear hybrid systems with continuous-state-dependent transitions: An imm approach. Aerospace and Electronic Systems, IEEE Transactions on 2009; 45(1): 376-392. DOI:10.1109/TAES.2009. 4805286 .

[15] Seah C and Hwang I. Stochastic linear hybrid systems: Modeling, estimation, and application in air traffic control. Control Systems Technology, IEEE Transactions on 2009; 17(3): 563-575. DOI:10.1109/TCST.2008.2001377. 
[16] Blom H and Bloem E. Exact bayesian and particle filtering of stochastic hybrid systems. Aerospace and Electronic Systems, IEEE Transactions on 2007; 43(1): 55-70. DOI: 10.1109/TAES.2007.357154.

[17] CAA. Air navigation: The order and regulations. Technical report, Civil Aviation Authority, 2015.

[18] FAA. Advisory circular: Recommended standard traffic pattens and practices for aeronautical operations at airports with operating control towers. Technical report, FAA, 1993.

[19] CAA. Guide to visual flight rules in the uk. Technical report, Civil Aviation Authority, 2011.

[20] CASA. Operations in the vicinity of non-controlled aerodromes. Technical report, Australian Government Civil Aviation Safety Authority, 2014. CAAP 166-1(3).

[21] Li X and Jilkov V. Survey of maneuvering target tracking. part i. dynamic models. Aerospace and Electronic Systems, IEEE Transactions on 2003; 39(4): 1333-1364. DOI: 10.1109/TAES.2003.1261132.

[22] Zhang Y and Bar-Shalom Y. Tracking move-stop-move targets with state-dependent mode transition probabilities. Aerospace and Electronic Systems, IEEE Transactions on 2011; 47(3): 2037-2054. DOI:10.1109/TAES.2011.5937281.

[23] Shao X, Huang B and Lee JM. Constrained bayesian state estimation a comparative study and a new particle filter based approach. Journal of Process Control 2010; 20(2): 143 - 157. DOI:http://dx.doi.org/10.1016/j.jprocont.2009.11.002. 